\title{
Adjuvant treatment of colorectal cancer in the elderly: Where do we come from and where are we going?
}

\author{
Maria Di Bartolomeo, Filippo Pietrantonio, Pamela Biondani, Filippo de Braud \\ Medical Oncology Unit 1, Fondazione IRCCS Istituto Nazionale dei Tumori, Milan, Italy \\ Correspondence: Maria Di Bartolomeo. Address: Medical Oncology Unit 1, Fondazione IRCCS Istituto Nazionale dei, \\ Tumori, Via Venezian, 1-20133 Milan, Italy. Telephone: 39-022-3902-882. Fax: 39-022-3902-149. \\ E-mail: maria.dibartolomeo@istitutotumori.mi.it
}

Received: January 26, 2012

Accepted: May 1, 2012

Published: June 1, 2012

DOI : $10.5430 /$ jst.v2n3p38

URL: http://dx.doi.org/10.5430/jst.v2n3p38

\section{Abstract}

Objective: Colorectal cancer is the third most commonly reported cancer in the world and about $50 \%$ of patients are diagnosed over the age of 70 years. The authors discuss age-related changes in organ function, comorbidities and frailty in the elderly, and their impact on chemotherapy toxicity.

Methods: The authors review data from observational studies and subgroup analyses of randomized clinical trials on adjuvant chemotherapy in elderly colorectal cancer patients.

Results: Several large population-based studies suggest that adjuvant chemotherapy is offered less frequently to elderly patients, although in recent years the prescription patterns tended to significantly increase. In fact, data from retrospective analyses of randomized trials indicate that elderly stage III colorectal cancer patients may get similar clinical advantage from adjuvant treatment with fluoropyrimidines, although major comorbidities may substantially limit life expectancy and minimize the survival benefits. The use of oxaliplatin-based regimens needs to take into account the individual risk/benefit profile due to lack of unequivocal evidence of positive literature data.

Conclusions: Adjuvant chemotherapy of colorectal cancer should be investigated by prospective trials specifically designed for the elderly. Fit elderly patients should be offered standard adjuvant treatments, while modified schedule, attenuated doses or even treatment omission can be offered to more frail patients.

\section{Key words}

Adjuvant chemotherapy, Elderly, Colorectal cancer

\section{I ntroduction}

\section{Colorectal cancer and the elderly}

Colorectal cancer (CRC) is the second most common cause of death from cancer in both men and women in Europe and is the third most commonly reported cancer in the world ${ }^{[1]}$. In 2006, CRC was the second most common form of cancer diagnosed in Europe, with a total of 412900 new cases and 207400 deaths. In particular, stage III CRC affects approximately 33000 elderly patients annually in the U.S. ${ }^{[2]}$. 
The incidence of CRC increases continuously with advancing age. Currently, the median age at diagnosis of CRC in the U.S. is 72 years old and approximately $50 \%$ of CRC patients are $\geq 70$ years old ${ }^{[3]}$. As the demographic proportion of elderly individuals continues to grow, the medical and social burden of CRC will increase over coming years.

The age limit currently accepted for the elderly definition is 70 years, although chronologic age is generally an imperfect surrogate for biological age. Ageing is associated with progressive decline of multiple organ functions, with an increased prevalence of comorbidity conditions. These changes influence treatment decision-making due to decrease of life expectancy and tolerance to chemotherapy. The best estimates of individual functional reserve may be provided by a comprehensive geriatric assessment (CGA), which takes into account functional and psychological status, comorbidities, socioeconomic conditions, pharmacy, cognition, nutrition and presence of specific geriatric syndromes. The assessment of function needs to include Activities of Daily Living (ADL) and Instrumental ADL (IADL) in addition to performance status and comorbidities indexes such as Cumulative Illness Rating Scale-Geriatrics (CIRS-G) and Charlson's Scale ${ }^{[4]}$. Not surprisingly, this index was independently correlated with chemotherapy tolerance in elderly CRC patients ${ }^{[5]}$. In fact, comobidities can constitute the most important prognostic factor for survival and minimize the benefits of adjuvant treatments. According to Balducci and Extermann ${ }^{[6]}$, CGA can identify three categories of older cancer patients. Group 1 includes patients without serious comorbidity, who are functionally independent and should be treated as younger counterparts; they typically qualify as good candidates for clinical trials and are therefore assessed by use of Eastern Cooperative Oncology Group (ECOG) Performance Scale; group 2 includes frail patients, candidates for supportive care alone; group 3 lies in between and identify the majority of elderly patients who display a limited number of IADL dependences and comorbidities; they may potentially benefit from medical treatment with adequate schedules and doses, although each case should be assessed by a multidisciplinary team on individual basis.

\section{Pharmacokinetics of fluoropyrimidines and oxaliplatin in the elderly}

Age-related pharmacokinetics changes encompass decreased excretion and metabolism of drugs. Thus, agents used in CRC adjuvant treatment could display an increased rate of adverse events (AEs). 5-fluorouracil (5-FU) dosage is often reduced arbitrarily, although there is no pharmacokinetic basis for dose modification based on age alone. Published data give conflicting results as to whether intravenous fluoropyrimidines are more toxic in the elderly. In a metanalysis of six randomised clinical trials (RCTs) ${ }^{[7]}$, older and poor performance status patients treated with 5-FU had higher incidence of non-haematological toxicity. Conversely, in the Intergroup 0089 study with adjuvant 5-FU, a secondary analysis demonstrated that the elderly are as likely to tolerate chemotherapy as younger patients ${ }^{[8]}$. In the metastatic setting, a retrospective analysis of European trials showed that fit elderly patients experience similar benefit and toxicity from 5-FU ${ }^{[9]}$. A main determinant of this controversy depends on the use of different schedules: Infusional 5-FU displays a more favorable toxicity profile than bolus administration, with severe hematological toxicity seven times less frequent ${ }^{[7]}$.

Capecitabine is an oral fluoropyrimidine which is metabolized to 5-FU and is generally well tolerated ${ }^{[10]}$. Thus this agent seems to be attractive in the elderly population due to side effects manageability and treatment compliance, with fewer hospital visits and no central venous catheters-related complications. The pharmacokinetics of capecitabine is not affected by age in patients with normal renal function, although patients with moderate renal impairment experience a higher incidence of severe AEs ${ }^{[11]}$. Therefore, International Society of Geriatric Oncology Chemotherapy (SIOG) taskforce recommends a lower starting dose in patients with creatinine clearance $(\mathrm{CrCl})$ of $30-50 \mathrm{~mL} / \mathrm{min}$, with absolute contraindication in patients with severely impaired $\mathrm{CrCl}(<30 \mathrm{~mL} / \mathrm{min})^{[12]}$.

Renal impairment decreases plasma ultrafiltrate platinum clearance and enhances systemic platinum exposure in cancer patients in cancer patients treated with oxaliplatin. However, in studies of patients with mild to moderate renal failure, no increased toxicities were observed. These observations are consistent with the hypothesis that the active forms of oxaliplatin are eliminated within the first hour after infusion by clearance mechanisms independent of renal function. During the later phase the inactive low-molecular-weight platinum is excreted via glomerular filtration which may explain 
why renal impairment does not enhance oxaliplatin related toxicities; thus, there are no data to support dose reduction based on chronologic age alone or renal impairment ${ }^{[13]}$.

\section{Literature search methodology}

The evidence regarding the tolerability and efficacy of adjuvant treatment of CRC in the elderly derives from two different sources: retrospective analyses of subgroups enrolled in randomized clinical trials (RCTs) without an upper age limit and prospective studies specifically designed to include elderly populations. For this article, the PubMed database was searched for articles on adjuvant chemotherapy published before January 2012; early release publications were also included. The search terms were 'adjuvant' AND ‘chemotherapy' AND ‘colorectal cancer' AND ‘elderly. Bibliographies of eligible studies' were also reviewed to identify additional studies, while data from abstract were not included.

\section{Results}

\section{Disparities of adjuvant treatment indication in the elderly}

Older patients are underrepresented in RCTs and this has led to a paucity of data available to the community oncologists to drive evidence-based treatment decisions; thus, treatment benefit to older patients and safety issues are often undetermined.

A study of Southwest Oncology Group clinical trials active between 1993 and 1998 found that although 63\% of US cancer patients were over $65 \%$ years old, the elderly comprised only $25 \%$ of trial participants ${ }^{[14]}$. Expanding the analysis to all cooperative groups and focusing on the later year (From 1997 to 2000), Lewis et al. found out that 32\% of patients cancer trials were age 65 years or older, consisting in a larger proportion of clinical trial participants than previously reported ${ }^{[15]}$.

Moreover, several studies have shown that older patients with stage II/III CRC receive a suboptimal adjuvant chemotherapy than younger ones. Possible reasons of treatment disparities include comorbidities, higher refusal rates, declining of functional and mental status, hospital volume and socioeconomic factors. Both physicians and patients may perceive a lowering of treatment efficacy and tolerance with advancing age ${ }^{[16]}$.

In a population-based study that considered 577 CRC patients aged 65-79 and diagnosed between 1995 and 2001 in the Eindhoven Cancer Registry of South Netherlands, adjuvant chemotherapy administration decreased with advancing age ( $80 \%$ of patients between $65-69$ versus $28 \%$ of patients aged $75-79$ ). Nevertheless, it rose significantly over time from $1995(19 \%)$ to 2001 (50\%), probably reflecting increasing amounts of supporting data in the literature ${ }^{[17]}$.

The "Oregon State Cancer Registry" (OSCaR) retrospective study identified 3789 CRC patients aged $\geq 80$ (29.5\% of total) ${ }^{[18]}$. It was shown that octogenarians (the so called "very elderly") received chemotherapy less frequently for every stage of disease, generally with single-agent.

As to whether there has been any change in practice patterns in the past decades, the evidence is conflicting. Schrag et al. ${ }^{[19]}$. studied the relationship between adjuvant chemotherapy use and age in a large cohort of patients from population-based registries (Surveillance, Epidemiology, and End Results/Medicare-linked database) collected between 1991 and 1996; the Authors identified 6262 stage III CRC patients aged $\geq 65$ and highlighted an extensive use in older patients (78\% in age group $65-69,74 \%$ in $70-74)$, although treatment rates declined dramatically after 75 years $(58 \%$ in $75-79,11 \%$ in $80-84)$. In this retrospective analysis, age still remained the strongest predictor of chemotherapy use after adjustment for confounding factors, such as comorbidities. A subsequent population-based SEER registry study on 8374 patients failed to show any significant sign of diminishing treatment disparity according to age over the time period spanning 1997-2002 ${ }^{[20]}$. 


\section{Adjuvant treatment efficacy in the elderly: Observational studies}

An increasing amount of observational studies has addressed the question if adjuvant treatment may improve survival endpoints in elderly CRC patients. Two separate cohort studies from the SEER/Medicare population-based registries spanning the mid 90's showed positive results. Iwashyna and Lamont ${ }^{[21]}$ performed a prospective, population-based cohort study of 3357 elderly patients with stage III CRC. Statistical analysis demonstrated a 5-year overall survival (OS) improvement with 5 -FU from $40.7 \%$ in untreated subjects to $52.7 \%$ in the treated population, with $27 \%$ reduction of death risk across 6 years of follow up and statistical significance maintained in octogenarians. In the second study from SEER/Medicare registries, Sunararajan et al. reported a hazard ratio (HR) of 0.66 favoring adjuvant treatment in the elderly ${ }^{[22]}$.

A more recent SEER/Medicare analysis from 1997 to 2002 included 6711 stage III CRC patients aged $\geq 66$ years. Notably, increasing age was inversely correlated with the use of adjuvant chemotherapy and the magnitude of treatment-related survival gain ${ }^{[23]}$.

Jessup et al. ${ }^{[24]}$ performed a large prospective study on the National Cancer Data Base between 1990 and 2002, including 85934 patients with stage III CRC (Around 25\% were octogenarians). Authors demonstrated that adjuvant chemotherapy use increased during time between $1991(39 \%)$ to $2002(64 \%)$, but still remained significantly lower in the elderly $(80 \%$, $70 \%$ and $40 \%$ in patients of $<70$ years, $70-79$ years and $\geq 80$ ). Nevertheless, adjuvant treatment of older patients conferred a survival benefit as much as younger ones, with a $16 \%$ improvement of 5 -year OS.

Despite these evidences, it should be pointed out that observational studies cannot provide definitive demonstration that adjuvant therapy improves survival in the elderly, because there is not full control for the distribution of important covariates among treatment groups.

On the other hand, Neogut et al. showed that nearly $30 \%$ of stage III CRC patients aged $\geq 65$ discontinued earlier 5 -FU-based adjuvant treatment ${ }^{[25]}$. Moreover, patients receiving $<5$ months of treatment had mortality rates nearly twice higher.

Kahn et al. reported an analysis on 705 stage III CRC patients enrolled in the CanCORS observational cohort study and assessed for important clinical morbid events. The Researchers showed that elderly patients aged $\geq 70$ were treated less frequently with adjuvant chemotherapy ( $58 \%$ versus $84 \%$ ), but experience similar rates of significant morbid events, even after adjustment for dose reduction and treatment duration ${ }^{[26]}$.

\section{Adjuvant treatment efficacy in the elderly: Randomized clinical trials}

The major evidence regarding adjuvant treatment of CRC might derive from two different sources: retrospective analyses of age subgroups enrolled into RCTs and prospective studies specifically designed for elderly. It is possible to argue that when clinical studies are designed to test the efficacy of a treatment intended for younger patients, only a selected proportion of older patients is considered for enrollment, without representation of the general elderly population. Moreover, the safety and efficacy of specific chemotherapy agents and combinations was not assessed in phase III RCT focusing primarily on the elderly.

\section{Fluoropyrimidines}

In 1990, bolus 5-FU became the standard adjuvant treatment for stage III CRC and the control regimen for consequent phase III trials, demonstrating a significant reduction of recurrence rate $(41 \%)$ and mortality $(33 \%){ }^{[27]}$. Sargent et al. ${ }^{[28]}$ conducted an age-based pooled analysis of individual patient data from seven phase III RCTs which compared 5-FU plus leucovorin (five trials) or plus levamisole (two trials) with surgery alone in stage II/III CRC. The total enrolment cohort was 3351 patients, $15.1 \%$ of whom were aged $\geq 70$. 5-FU chemotherapy conferred a $7 \%$ absolute improvement of 5 -year OS in both younger ( $71 \%$ vs. $64 \%$ ) and elderly patients (69\% vs. $62 \%)$, while increased 5 -year recurrence-free survival 
(RFS) from $58 \%$ to $69 \%$. No significant interaction was observed between age and treatment efficacy and safety. Age $\geq 70$ had no impact on recurrence risk but was prognostic for survival, as would be expected given the increased risk of dying from other causes. Regarding AEs, there was not higher incidence of nausea/vomiting, stomatitis or diarrhoea in the elderly; although leukopenia rate was significantly higher among those who received 5-FU plus levamisole, only a borderline increase was noted in the group treated with 5-FU and leucovorin. Octogenarians were only $0.7 \%$ of included patients, thus any conclusion about this age group should be drawn carefully. It should be emphasized that patients in this analysis were trial candidates; thus, the results can be weakened by selection bias, due to enrollment of patients with better performance status and minimal comorbidities as a result of screening. This could result in data with limited applicability to real-world patients.

A randomized, multicenter, phase III trial compared the efficacy and toxicity of adjuvant treatment with 12 weeks

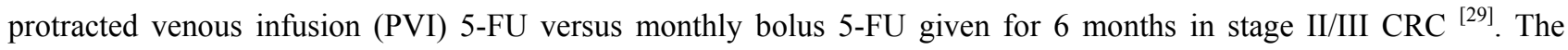
researchers enrolled a total of 801 patients and showed that PVI 5-FU was associated with a trend towards better RFS and OS compared with bolus 5-FU/LV, with significantly less toxicity. Consistent with the pooled analysis of RCTs, survival was similar in patients aged $\geq$ or $<70$ years. Based on these data, SIOG experts recommend infusional 5-FU as the best regimen in case of intravenous monotherapy, given the potential increase of toxicity with bolus regimens, particularly in the elderly.

The X-ACT phase III, randomized study compared adjuvant capecitabine versus bolus 5-FU/FA in 1987 stage III CRC patients, with an upper age limit of 75 years and a total enrollment of 397 elderly ${ }^{[30]}$. This non-inferiority study demonstrated that capecitabine conferred a DFS advantage at least equivalent to Mayo Clinic regimen (In the intention-to-treat analysis, $P<0.001$ for the comparison of the upper limit of the HR with the non-inferiority margin of 1.20 ), with a superiority of RFS (HR, $0.86 ; 95 \% \mathrm{CI}, 0.74$ to $0.99 ; P=0.04$ ). Aged subgroup analysis confirmed these efficacy results in patients aged $<$ and $\geq 70$ years. A more recent 5 -year update from this trial showed a trend towards superiority of capecitabine for 5-year OS (HR, $0.86 ; 95 \% \mathrm{CI}, 0.74-1.01 ; P=0.06)$. Nevertheless, age was an independent

poor prognostic factor for OS in the multivariate analysis ${ }^{[31]}$. Capecitabine demonstrated a favorable safety profile also in older patients, who experienced significantly less diarrhoea, stomatitis, nausea, vomiting, alopecia and neutropenia, although more reported grade 3 hand-foot syndrome.

\section{Oxaliplatin-based regimens}

The Multicenter International Study of Oxaliplatin/5-Fluorouracil/Leucovorin in the Adjuvant Treatment of Colon Cancer (MOSAIC) trial and the National Surgical Adjuvant Breast and Bowel Project (NSABP) C-07 trial demonstrated that adding oxaliplatin to a biweekly schedule of infused (MOSAIC) or weekly bolus (C-07) 5-FU/FA significantly prolongs DFS in stage II/III CRC, with relative risk reductions of $23 \%$ and $21 \%$, respectively.

The MOSAIC trial ${ }^{[32]}$ was a phase III, multicenter, randomized study whose primary endpoint was the demonstration of a $25 \%$ decrease of recurrence risk at 3 years among 2246 patients with stage II/III CRC receiving infusional 5-FU/FA alone (De Gramont regimen) or an oxaliplatin-based combination (FOLFOX-4). Adding oxaliplatin to 5-FU infusion and leucovorin significantly improved 3-year DFS from $72.9 \%$ to $78.2 \%$. In 2009 updated data in terms of DFS and OS were published: 5-year DFS was $73.3 \%$ in FOLFOX-4 group and 67.4\% in 5-FU/FA group (HR, 0.80; 95\% CI, 0.68 to 0.93 ; $P=0.003$ ); 6-year OS was $78.5 \%$ and $76 \%$, respectively (HR, $0.84 ; 95 \% \mathrm{CI}, 0.71$ to $1.00 ; P=0.046$; considering stage III patients subgroup: HR, $0.80 ; 95 \% \mathrm{CI}, 0.65$ to $0.97 ; P=0.023$ ). No differences in DFS and OS were found in stage II; although a trend towards improved 5-years DFS was observed in the subgroup of patients with high-risk node-negative disease ${ }^{[33]}$.

In the MOSAIC trial, median age 61 years and 463 patients were aged $\geq 65$ (35.6\% in FOLFOX-4 arm, 33.8\% in 5-FU/FA arm). An age subgroup analysis showed that the efficacy of FOLFOX-4 vs. 5-FU/FA in terms of OS was not maintained in patients older than 65 years ${ }^{[32]}$. Older patients probably received short-term DFS benefit from FOLFOX-4 that 
disappeared with longer follow-up, due to deaths from competing causes. Researchers found no difference in dose intensity between older and younger patients, although $5 \%$ fewer elderly patients completed the scheduled 12 courses of chemotherapy.

Pooled data analysis of 3742 CRC patients from 4 RCTs testing FOLFOX-4 as adjuvant, first-line or second-line setting led to collection of efficacy and safety data in the elderly population $(16 \%)^{[34]}$. It was stated that grade $\geq 3$ hematologic toxicity (neutropenia and thrombocytopenia) and fatigue were significantly higher in age group $\geq 70$ years. Nevertheless, there were not age-related differences in nausea/vomiting, diarrhoea, severe neurotoxicity and overall incidence of severe AEs. However, we previously pointed out that elderly patients enrolled into clinical trials fulfill strict inclusion criteria and are obviously selected for their "fitness" and absence of severe comorbidities. Thus, some older CRC patients may not benefit and tolerate aggressive adjuvant treatment with newer drugs, beyond 5-FU/FA, particularly regarding oxaliplatin-based combinations.

McCleary et al. ${ }^{[35]}$ presented data from the ACCENT (Adjuvant Colon Cancer End Points) database on 2170 CRC patients aged $\geq 70$ and treated in 6 phase III adjuvant RCTs (MOSAIC, NSABP C-07, CALGB 89803, PETACC 03, X-ACT, and NSABP C-06). The investigators compared 5-FU monotherapy to oral fluoropyrimidines or to combination regimens with irinotecan/oxaliplatin. This retrospective metanalysis found that elderly patients did not receive a clear benefit from combination chemotherapy and newer adjuvant regimens in terms of DFS and OS. Even considering the lack of efficacy of irinotecan-based therapy and looking at the 2 oxaliplatin-based trials (C-07 and MOSAIC), these results was confirmed (HR, 1.04 for DFS, 1.19 for OS). Recently, a subgroup analysis of the "true" elderly patients aged $\geq 70$ years ( $\mathrm{n}=315)$ in the MOSAIC trial was reported, reviewing clinical characteristics and comorbidities. It was stated that HR for DFS was 0.91 (95\% CI, 0.62 to 1.34$)$ and HR for OS was 1.10 (95\% CI, 0.73 to 1.65). The Authors declared that DFS benefit of oxaliplatin-based adjuvant treatment was of less magnitude in the elderly. Lack of OS differences did not seem to be related to comorbidities, but possibly to different management/outcome of relapses or incidence of second cancers ${ }^{[36]}$.

The NO16968 trial (XELOXA) was an open-label, randomized, phase III study of XELOX (Oral capecitabine plus intravenous oxaliplatin) versus bolus 5-FU/FA as 6-months adjuvant therapy for stage III CRC ${ }^{[37]}$. The primary end point of the study, which enrolled 1886 patients, was to demonstrate the superiority of XELOX over 5-FU/FA in terms of DFS; secondary endpoints included OS, RFS, safety profiles and perceived treatment convenience. Patients in the XELOX group displayed significantly better outcome compared to those receiving 5-FU/FA (3-year DFS 70.8\% vs. 66.5\% and 5-year DFS $66.1 \%$ vs. $59.8 \%$ (HR, $0.8 ; 95 \%$ CI, 0.69 to $0.93 ; P=0.0045$ ). Although overall survival data are currently immature and longer follow up is needed, 5 -year OS for XELOX and 5-FU/FA was $77.6 \%$ and $74.2 \%$, respectively (HR, 0.87 ; 95\% CI, 0.72-1.05; $P=0.1486$ ).

Regarding severe AEs, XELOX caused less stomatitis, neutropenia and febrile neutropenia than 5-FU/FA, but more frequent hand-foot syndrome and thrombocytopenia. Sixty-day all-cause mortality was $1 \%$ on both arms. Authors concluded that XELOX is feasible in the adjuvant setting and compares favorably with FOLFOX-4 in terms of toxicity.

An age subgroup analysis showed that the 3-year DFS benefit for patients receiving XELOX versus 5-FU/FA was maintained in elderly patients ${ }^{[38]}$. In patients aged $\geq 70$ years $(\mathrm{n}=409)$, DFS was $66 \%$ in the XELOX arm versus $60 \%$ in the 5-FU/FA arm (HR 0.87; 95\% CI 0.63 to 1.18). In patients aged $\geq 65$ years ( $\mathrm{n}=744$ ), the respective results were $68 \%$ and $62 \%$ (HR $0.81 ; 95 \%$ CI 0.64 to 1.03 ). However, neither result was statistically significant as the $95 \%$ CI of the hazard ratios crossed 1. Although overall survival data are immature, the analysis demonstrated a trend towards OS superiority for XELOX over 5-FU/FA in patients aged $\geq 65$ ( $73 \%$ versus $70 \%)$ and $\geq 70$ (69\% vs. $67 \%$; HR, $0.94 ; 95 \%$ CI, 0.66 to 1.34 ). Overall, these findings may differ from those of the MOSAIC study and the ACCENT analysis. However, it must be pointed out that dose intensity and treatment duration were significantly lower in patients older than 70 years. In XELOX arm, incidence of severe AEs differed significantly in younger versus elderly patients (57\% vs. $70 \%$ ), particularly regarding grade $3 / 4$ diarrhoea ( $18 \%$ vs. $26 \%)^{[39]}$. Based on these data, XELOX is currently considered a promising 
regimen for adjuvant treatment of fit elderly patients with stage III CRC, although not yet included in SIOG expert recommendations.

In a recent cohort U.S. study examining 2560 patients, it was stated that older age was significantly associated with omission of oxaliplatin in postoperative chemotherapy and treatment duration $<3$ months ${ }^{[40]}$. When oxaliplatin was added to capecitabine, a two-fold increased likelihood of treatment discontuinuation was observed, probably due to toxicity. In fact, a randomized phase III trial comparing XELOX versus FOLFOX-4 in the metastatic setting reported a higher rate of severe gastrointestinal toxicity and hand-foot syndrome with the use of capecitabine ${ }^{[41]}$.

\section{Conclusions and future directions}

Standard adjuvant treatment of CRC in elderly patients is a controversial topic because of the lack of specific prospective data. The existing evidence suggests that elderly stage III CRC patients get similar clinical benefit from adjuvant treatment, although the advantages deriving from oxaliplatin-based regimens is less than younger patients. Moreover, the toxicity exacerbation (Particularly with newer oxaliplatin-based combinations) still constitutes the major issue. Careful monitoring for AEs and rapid intervention with supportive measures when toxicity occurs is a mandatory component of optimal care. Taking in mind that age alone should not drive clinical decision-making, elderly patients should be evaluated for adjuvant treatment on an individual basis and by a multidisciplinary team through a CGA. When chemotherapy is medically appropriate, patient preferences need to be weighed, along with individual functional reserve and life expectancy. It is important to establish an overall treatment plan for the management of elderly CRC patients, even if the major issue is to effectively select patients suitable for standard or attenuated therapy. Fit elderly patients should be offered standard adjuvant treatments, although the use of oxaliplatin needs to take into account the individual risk/benefit profile in presence of controversial literature data. Survival benefit may be important for some patients, and toxicity is manageable with attention to the increased risk of myelosuppression in the elderly. Less toxic options or even treatment omission can be offered to more frail patients.

\section{References}

[1] Ferlay J, Autier P, Boniol M et al. Estimates of the cancer incidence and mortality in Europe in 2006. Ann Oncol 2007;18:581-92. PMid:17287242 http://dx.doi.org/10.1093/annonc/mdl498

[2] Ries LAG, Harkins D, KrapchoM Contents of the SEER Cancer Statistics Review, 1975-2003 2003 Accessed September 27, 2006 Bethesda, MD National Cancer Institute Available at http://seer.cancer.gov/csr/1975_2003/.

[3] World Health Organization. GBD 2001: Deaths by age, sex and cause for the year 2001. [On-line] http://www.who.int/mediacentre/news/releases/2003/pr27/en/

[4] Charlson ME, Pompei P, Ales KL, et al. A new method of classifying prognostic comorbidity in longitudinal studies: Development and validation. J Chronic Dis 1987;40:373-83. http://dx.doi.org/10.1016/0021-9681(87)90171-8

[5] Aparicio T, Desrame J, Lecomte T, et al. Oxaliplatin- or irinotecan-based chemotherapy for metastatic colorectal cancer in the elderly. Br J Cancer 2003;89:1439-44. PMid:14562014 http://dx.doi.org/10.1038/sj.bjc.6601310

[6] Balducci L, Extermann M. Management of cancer in the older person: A practical approach. The Oncologist 2000;5:224-37. PMid:10884501 http://dx.doi.org/10.1634/theoncologist.5-3-224

[7] Meta-Analysis Group in Cancer: Toxicity of fluorouracil in patients with advanced colorectal cancer: Effect of administration schedule and prognostic factors. J Clin Oncol 1998;16:3537-41. PMid:9817272

[8] Haller DG, Catalano PJ, MacDonald JS, et al. Phase III study of fluorouracil, leucovorin, and levamisole in high-risk II and III colon cancer: Final report of Intergroup 0089. J Clin Oncol 2005;23:8671-78. PMid:16314627 http://dx.doi.org/10.1200/JCO.2004.00.5686

[9] Folprecht G, Cunningham D, Ross P, et al. Efficacy of 5-fluorouracil-based chemotherapy in elderly patients with metastatic colorectal cancer: A pooled analysis of clinical trials. Ann Oncol 2004;15:1330-38. PMid:15319237

http://dx.doi.org/10.1093/annonc/mdh344 
[10] Cassidy J, Twelves C, Cameron D, et al. Bioequivalence of two tablet formulations of capecitabine and exploration of age, gender, body surface area, and creatinine clearance as factors influencing systemic exposure in cancer patients. Cancer Chemother Pharmacol 1999;44:453-60. PMid:10550565 http://dx.doi.org/10.1007/s002800051118

[11] Cassidy J, Twelves C, Van Cutsem E, et al. First-line oral capecitabine therapy in metastatic colorectal cancer: A favorable safety profile compared with intravenous 5-fluorouracil/leucovorin. Ann Oncol 2002;13:566-75. PMid:12056707 http://dx.doi.org/10.1093/annonc/mdf089

[12] Lichtman SM, Wildiers H, Chatelut E, et al. International Society of Geriatric Oncology Chemotherapy Taskforce: Evaluation of Chemotherapy in Older Patients - An Analysis of the Medical Literature. J Clin Oncol 2007;25:1832-43. PMid:17488981 http://dx.doi.org/10.1200/JCO.2007.10.6583

[13] Takimoto CH, Graham MA, Lockwood G et al. Oxaliplatin pharmacokinetics and pharmacodynamis in adult cancer patients with impaired renal function. Clinical Cance research 2007;13:4832-39.

[14] Hutchins LK, Unger JM, Crowed JJ, et al. Underrepresentation of patients 65 years of age or older in cancer-treatment trials. N Engl J Med 1999;341:2061-67. PMid:10615079 http://dx.doi.org/10.1056/NEJM199912303412706

[15] Lewis JH, Kilgore ML, Goldman DP, et al. Partecipation of patients 65 years of age or older in cancer clinical trials. J Clin Oncol 2003;21:1383-89. PMid:12663731 http://dx.doi.org/10.1200/JCO.2003.08.010

[16] Aparicio T, Navazesh A, Boutron I, et al. Half of elderly patients routinely treated for colorectal cancer receive a sub-standard treatment. Crit Rev Oncol Hematol 2009;71:249-57. PMid:19131256 http://dx.doi.org/10.1016/j.critrevonc.2008.11.006

[17] Lemmens VEPP, Van Halteren AH, Janssen-Heijnen MLG, et al. Adjuvant treatment for elderly patients with stage III colon cancer in the southern Netherlands is affected by socioeconomic status, gender, and comorbidity. Ann Oncol 2005;16:767-72. PMid:15817594 http://dx.doi.org/10.1093/annonc/mdi159

[18] Hardiman KM, Cone M, Sheppard BC, et al. Disparities in the treatment of colon cancer in octogenarians. Am J Surg 2009; 197:624-628. PMid:19393356 http://dx.doi.org/10.1016/j.amjsurg.2008.12.018

[19] Schrag D, Cramer LD, Bach PB, et al. Age and adjuvant chemotherapy use after surgery for stage III colon cancer. J Natl Cancer Inst 2001;93:850-57. http://dx.doi.org/10.1093/jnci/93.11.850

[20] Davidoff AJ, Choti MA, Zuckerman IH, et al. Is there evidence of diminishing disparities in treatment with adjuvant chemotherapy among elderly stage 3 colon cancer patients? An analysis of 8,374 patients from SEER-Medicare data. ASCO Annual Meeting Proceedings. J Clin Oncol 2008; 26 (May 20 suppl; abstr 6574).

[21] Iwashyna TJ, Lamont EB. Effectiveness of adjuvant fluorouracil in clinical practice: A population-based cohort study of elderly patients with stage III colon cancer. J Clin Oncol 2002;20:3992-98. PMid:12351596 http://dx.doi.org/10.1200/JCO.2002.03.083

[22] Sundararajan V, Mitra N, Jacobson JS, et al. Survival associated with 5-fluorouracil-based adjuvant chemotherapy among elderly patients with node-positive colon cancer. Ann Intern Med 2002;136:349-57. PMid:11874307

[23] Zuckerman IH, Davidoff AJ, Onukwugha E, et al. Effect of age on survival benefit of adjuvant chemotherapy in elderly stage III colon cancer patients: A population-based analysis. ASCO Annual Meeting Proceedings. J Clin Oncol 2008;26 (May 20 suppl; abstr 4014).

[24] Jessup JM, Stewart A, Greene FL, et al.: Adjuvant chemotherapy for stage III colon cancer: Implication of race/ethnicity, age and differentiation. JAMA 2005;294:2703-11. PMid:16333005 http://dx.doi.org/10.1001/jama.294.21.2703

[25] Neugut AI, Matasar M, Wang X, et al. Duration of adjuvant chemotherapy for colon cancer and survival among the elderly. J Clin Oncol 2006;24:2368-75. PMid:16618946 http://dx.doi.org/10.1200/JCO.2005.04.5005

[26] Kahn KL, Adams JL, Chrischilles EE, et al. Are we using of chemotherapy for elderly stage III colon cancer patients? An analysis from the Cancer Care outcomes \& Research Surveillance Consortium (CanCORS). ASCO Annual Meeting Proceedings. J Clin Oncol 2008;26 (May 20 suppl; abstr 6500).

[27] Moertl CG, Fleming TR, MacDonald JS, et al. Levamisole and fluorouracil for adjuvant therapy of resected colon carcinoma. N Engl J Med 1990;322:352-58. PMid:2300087 http://dx.doi.org/10.1056/NEJM199002083220602

[28] Sargent DJ, Goldberg RM, Jacobson SD, et al. A pooled analysis of adjuvant chemotherapy for resected colon cancer in elderly patients. N Engl J Med 2001;345:1091-97. PMid:11596588 http://dx.doi.org/10.1056/NEJMoa010957

[29] Chau I, Norman AR, Cunningham D, et al. A randomised comparison between 6 months of bolus fluorouracil/leucovorin and 12 weeks of protracted venous infusion fluorouracil as adjuvant treatment in colorectal cancer. Ann Oncol 2005;16:549-57. PMid:15695501 http://dx.doi.org/10.1093/annonc/mdi116

[30] Twelves C, Wong A, Nowacki Mp et al. Capecitabine as adjuvant treatment for stage III colon cancer. N Engl J Med 2005;352:2696-704. PMid:15987918 http://dx.doi.org/10.1056/NEJMoa043116

[31] Twelves C, Scheithauer W, McKendrik J, et al. Capecitabine versus 5-fluorouracil/folinic acid as adjuvant therapy for stage III colon cancer: Final results from the X-ACT trial with analysis by age and preliminary evidence of a pharmacodynamic marker of efficacy. Ann Oncol 2011; [publication ahead of print] 
[32] André T, Boni C, Mounedji-Boudiaf L, et al. Oxaliplatin, fluorouracil, and leucovorin as adjuvant treatment for colon cancer. N Engl J Med 2004;350:2343-51. PMid:15175436 http://dx.doi.org/10.1056/NEJMoa032709

[33] André T, Boni C, Navarro M, et al. Improved overall survival with oxaliplatin, fluorouracil, and leucovorin as adjuvant treatment in stage II or III colon cancer in the MOSAIC trial. J Clin Oncol 2009;27:3109-16. PMid:19451431 http://dx.doi.org/10.1200/JCO.2008.20.6771

[34] Goldberg RM, Tabah-Fisch I, Bleiberg H, et al. Pooled analysis of safety and efficacy of oxaliplatin plus fluorouracil/leucovorin administered bimonthly in elderly patients with colorectal cancer. J Clin Oncol 2006;24:4085-91. PMid:16943526 http://dx.doi.org/10.1200/JCO.2006.06.9039

[35] McCleary NA, Meyerhardt J, Green E, et al. Impact of older age on the efficacy of newer adjuvant therapies in $>12,500$ patients with stage II/III colon cancer: Findings from the ACCENT database. ASCO Annual Meeting Proceedings, J Clin Oncol 2009;27:15s (suppl; abstr 4010).

[36] Tournigand C, André T, Bachet J, et al. FOLFOX4 as adjuvant therapy in elderly patients (pts) with colon cancer (CC): Subgroup analysis of the MOSAIC trial. ASCO Annual Meeting Proceedings. J Clin Oncol 2010;28:15s (suppl; abstr 3522). PMid:21383294 http://dx.doi.org/10.1200/JCO.2010.33.6297

[37] Daniel G. Haller, Josep Tabernero, Jean Maroun, et al. Capecitabine Plus Oxaliplatin Compared With Fluorouracil and Folinic Acid As Adjuvant Therapy for Stage III Colon Cancer. J Clin Oncol 2011;29:1465-71.

[38] Haller DG, Cassidy J, Tabernero J, et al. Efficacy findings from a randomized phase III trial of capecitabine plus oxaliplatin versus bolus 5-FU/LV for stage III colon cancer (NO16968): Impact of age on disease-free survival (DFS). ASCO Annual Meeting Proceedings. J Clin Oncol 2010;28:15s (suppl; abstr 3521).

[39] Schmoll HJ, Cartwright T, Tabernero J, et al: Phase III trial of capecitabine plus oxaliplatin as adjuvant therapy for stage III colon cancer: A planned safety analysis in 1,864 patients. J Clin Oncol 2007;25:102-109. PMid:17194911 http://dx.doi.org/10.1200/JCO.2006.08.1075

[40] Abrams TA, Brightly R, Mao J, et al. Patterns of adjuvant chemotherapy use in a population-based cohort of patients with resected stage II or III colon cancer. J Clin Oncol 2011;29:3255-62. PMid:21768462 http://dx.doi.org/10.1200/JCO.2011.35.0058

[41] Cassidy J, Clarke S, Diaz-Rubio E, et al. XELOX-1/NO16966, a randomized phase III trial of first-line XELOX compared with FOLFOX4 for patients with metastatic colorectal cancer (MCRC): Updated survival and tolerability results. ASCO Gastrointestinal Cancers Symposium 2009; abstr 382. 